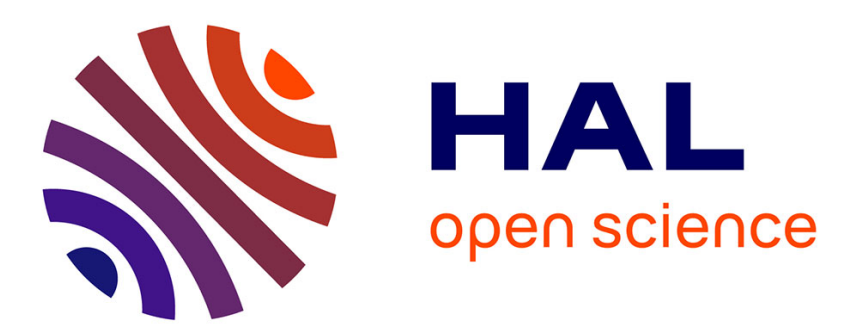

\title{
Étude des convertisseurs multicellulaires parallèles : I. Modélisation
}

Pascal Davancens, Thierry Meynard

\section{To cite this version:}

Pascal Davancens, Thierry Meynard. Étude des convertisseurs multicellulaires parallèles: I. Modélisation. Journal de Physique III, 1997, 7 (1), pp.143-160. 10.1051/jp3:1997116 . jpa-00249566

\section{HAL Id: jpa-00249566 https://hal.science/jpa-00249566}

Submitted on 1 Jan 1997

HAL is a multi-disciplinary open access archive for the deposit and dissemination of scientific research documents, whether they are published or not. The documents may come from teaching and research institutions in France or abroad, or from public or private research centers.
L'archive ouverte pluridisciplinaire HAL, est destinée au dépôt et à la diffusion de documents scientifiques de niveau recherche, publiés ou non, émanant des établissements d'enseignement et de recherche français ou étrangers, des laboratoires publics ou privés. 


\title{
Étude des convertisseurs multicellulaires parallèles : I. Modélisation
}

\author{
Pascal Davancens et Thierry Meynard (*)
}

Laboratoire d'Électrotechnique et d'Électronique Industrielle $\left(^{* *}\right)$, I.N.P. Toulouse - E.N.S.E E.I.H.T., 2 rue Charles Camichel, 31071 Toulouse Cedex, France

(Reçu le 13 mai 1996, révisé le 25 septembre 1996, accepted le 8 octobre 1996)

PACS.02.60.Cb - Numerical simulation; solution of equation

PACS.84.30.JC - Power electronics, power supply circuits

PACS.84.70.+P - High-current and high-voltage technology: power systems

Résumé. - Dans le domaine des forts courants avec des fréquences de découpage élevées, de nouvelles structures basées sur l'association de composants ont été développées. Parmi elles, nous trouvons les convertisseurs multicellulaires parallèles que nous présentons dans cet article Une particularıté de ces structures est leur capacité à assurer un équilibrage naturel des courants dans les divers interrupteurs. Dans un premier temps, à l'aide d'un exemple simple, nous montrons que ce phénomène est assuré par des harmonıques du courant d'entrée générées par le convertisseur en cas de déséquilibre. Cependant, pour un nombre important de cellules de commutation en parallèle, la complexité du système rend impossible toute prédiction du comportement transitoire du convertisseur et par conséquent tout prédimensionnement Dans un deuxième temps, nous proposons une modélisation basée sur une décomposition harmonıque des courants afin d'identifier les mécanısmes de rééquilibrage que nous validons à l'aide d'un logiciel de simulation de convertisseurs statiques.

\begin{abstract}
In the field of high current power conversion with high switching frequency, new topologies using parallel devices have been introduced, among which parallel multicell converters. An intrinsic feature of these converters is natural balancing of the currents flowing through the different switches. In the first section, an example will be used to demonstrate that this natural balancing property is related to the extra current harmonics induced by the current unbalance. However, for higher numbers of cells in parallel, the increased complexity does not allow predicting the transient behaviour of the system which makes design difficult. In the second section, a model based on the harmonic analysis is proposed to determine the balancing mechanisms; this model is then checked by simulation
\end{abstract}

( ${ }^{*}$ Auteur auquel dort être adressée la correspondance (e-mall : meynard@leei.enseerht.fr)

$\left({ }^{* *}\right)$ U.P.R.E.S. - A au C.N.R S. N 5004

(C) Les Éditions de Physique 1997 


\section{Notations}

\begin{tabular}{|c|c|}
\hline$p$ & Nombre de cellules en parallèle \\
\hline$k$ & Indice de numérotation des cellules de commutation \\
\hline$r$ & Nombre d'harmoniques pris en compte dans la modélisation \\
\hline$n$ & Indice de numérotation des harmoniques \\
\hline$F_{\mathrm{dec}}$ & Fréquence de découpage \\
\hline$A_{k}$ & Interrupteur du haut de la cellule $k$ \\
\hline$B_{k}$ & Interrupteur du bas de la cellule $k$ \\
\hline$c_{k}$ & Nœud de la cellule $k$ \\
\hline$V_{c_{k}}$ & Potentiel du point $c_{k}$ \\
\hline $\mathcal{R}_{k}$ & Rapport cyclique de la cellule $k$ \\
\hline $\mathcal{R}$ & Rapport cyclique lorsqu'il est égal dans toutes les cellules \\
\hline$\Phi_{k}$ & Déphasage de la commande de la cellule $k$ \\
\hline$L_{k}$ & Inductance de liaison de la cellule $k$ \\
\hline$L$ & Inductance de liaison lorsqu'elle est de meme valeur dans toutes les cellules \\
\hline$I_{L_{k}}$ & Courant dans l'inductance $L_{k}$ \\
\hline$\dot{I}_{L_{k}}$ & Dérivée du courant dans l’inductance $L_{k}$ \\
\hline$V_{L_{k}}$ & Tension aux bornes de l'inductance $L_{k}$ \\
\hline$\left(I_{\mathrm{tr}_{k}}\right)_{\text {on }}$ & Courant dans l'interrupteur passant de la cellule $k$ \\
\hline s & Noud de connexion des inductances de liaison \\
\hline$V_{\mathrm{s}}$ & Potentiel du point $s$ \\
\hline$I_{\mathrm{s}}$ & Courant de sortie du convertisseur \\
\hline$E$ & Source de tension d'entrée du convertisseur \\
\hline$I_{\mathrm{e}}$ & Courant fourni par la source $E$ \\
\hline$I_{\mathrm{e}}^{n}$ & Harmonique de rang $n$ du courant fourni par la source $E$ \\
\hline$R_{\mathrm{ch}}$ & Résistance de la charge du convertisseur \\
\hline$L_{\mathrm{ch}}$ & Inductance de la charge du convertisseur \\
\hline$V_{\mathrm{ch}}$ & F.e.m. de la charge du convertisseur \\
\hline$Z_{\mathrm{e}}$ & Impédance d'entrée du convertisseur \\
\hline$Z_{\mathrm{e}}^{n}$ & Valeur de l'impédance d'entrée à la fréquence $n F_{\text {dec }}$ \\
\hline$V_{\mathrm{e}}$ & Tension d'entrée du convertisseur \\
\hline$V_{e}^{n}$ & Harmonique de rang $n$ de la tension d'entrée \\
\hline$\Psi^{n}$ & Phase de $V_{\mathrm{e}}^{n}$ \\
\hline$\left\|V_{\mathrm{e}}^{n}\right\|$ & Norme de $V_{\mathrm{e}}^{n}$ \\
\hline$R_{\mathrm{ds}_{\mathrm{on}}}$ & Résistance passante d'un interrupteur \\
\hline$R_{\mathrm{on}_{h}}$ & Résistance passante d'un interrupteur de la cellule $k$ \\
\hline$H_{n}$ & Grandeur introduite relative à l'harmonique de rang $n$ \\
\hline$G_{k}^{n}$ & Grandeur introduite relative à la cellule $k$ et à l'harmonique de rang $n$ \\
\hline$V_{k}^{n}$ & Tension générée par $V_{\mathrm{e}}^{n}$ au nœud $c_{k}$ de la cellule $k$ \\
\hline $\bar{V}_{k}^{n}$ & Valeur moyenne de $V_{k}^{n}$ \\
\hline$X$ & Matrice d'état du système \\
\hline$\dot{X}$ & Dérivée de la matrice $X$ \\
\hline$X_{\mathrm{p}}$ & Matrice du régime permanent de $X$ \\
\hline$A$ & Matrice dynamique élémentaire du système \\
\hline$B$ & Matrice de commande élémentaire du système \\
\hline$A_{\mathrm{ch}}$ & Matrice dynamique du système avec prise en compte de la charge \\
\hline$B_{\mathrm{ch}}$ & Matrice de commande du système avec prise en compte de la charge \\
\hline
\end{tabular}


$\tilde{A}_{\text {ch }} \quad$ Matrice dynamique du système avec prise en compte des $R_{\mathrm{ds}_{\mathrm{on}}}$ et de la charge

$\tilde{B}_{\mathrm{ch}} \quad$ Matrice de commande du système avec prise en compte des $R_{\mathrm{ds} \text { on }}$ et de la charge

$U \quad$ Vecteur d'entrée du système

$M \quad$ Matrice introduite pour la prise en compte de la charge

$I_{p} \quad$ Matrice identité de dimension $p, p$

\section{Introduction}

D'une manière générale la modélisation en vue de la commande des convertisseurs statiques présente une difficulté majeure liée au fait que ces systèmes incluent des variables continues (courants dans les inductances, tensions aux bornes des capacités) et des variables discrètes (état passant ou bloqué des interrupteurs). Bien sûr, c'est au sens d'un modèle macroscopique que l'interrupteur possède ce caractère binaire. Par ailleurs, lors de son fonctionnement le convertisseur présente une topologie variable à caractère périodique. Ces remarques nous permettent d'envisager la mise à jour de deux types de modèle : soit aux valeurs moyennes, soit un modèle global où l'état de l'interrupteur apparaît par l'intermédiaire d'une fonction discrète dite de commutation.

Pour cette première catégorie il existe une méthodologie simple, lorsque les grandeurs continues peuvent être représentées par leur valeur moyenne sur une période de fonctionnement. Ceci est en général le cas, notamment pour les convertisseurs à MLI, qui présentent un filtre de sortie dimensionné afin de réduire les ondulations résiduelles. Cette approche a donné lieu à différentes méthodes de modélisation (circuit moyen équivalent, moyenne dans l'espace d'état...) [1] et aboutit à un modèle continu que l'on peut échantillonner.

La deuxième catégorie n'exige aucune restriction, mais le modèle généré est souvent complexe et de caractéristique non linéaire [2]. Bien que destiné à la simulation [3], son utilisation en commande est possible à l'aide d'un formalisme mathématique adapté [4]. Cependant, la linéarisation autour d'un point de fonctionnement, bien que fastidieuse, conduit à des applications intéressantes $[5,6]$.

L'obtention d'un modèle peut également être envisagé par le biais d'une décomposition harmonique au premier ordre des grandeurs d'état, ceci conduit à un modèle contınu représentant le convertisseur au sens du premer harmonique [7].

L'utilisation des convertisseurs statiques dans des domaines de puissance sans cesse croissants a donné lieu à des associations particulières de cellules de commutation permettant d'obtenir des caractéristiques de tenue en courant et en tension améliorées, c'est le cas des convertisseurs multicellulaires parallèle. Les exigences relatives au bon contrôle de ces structures passent nécessairement par une étape de modélisation dont le principe se doit d'être intimement lié à la topologie de ces nouvelles associations Une particularité de ces convertisseurs réside dans le fait que ce sont les harmoniques du courant d'entrée qui, bien que d'amplitudes très faibles devant la valeur moyenne, régissent l'évolution de l'état du convertisseur. En effet ces harmoniques assurent l'équilibrage naturel des courants dans les semi-conducteurs en modifiant la tension aux bornes des cellules de commutation. Devant cet état de fait, il apparait clairement que les modèles au sens des valeurs moyennes sont à proscrire et qu'il y a lieu de développer des modèles prenant en considération ces harmoniques.

Cette approche peut être menée à bien en choisissant une configuration quelconque du convertisseur et en remplaçant les interrupteurs fermés par des sources de courant intégrant les harmoniques et les interrupteurs ouverts par des sources de tensions représentant également les différents harmoniques. 


\section{Rappel sur les convertisseurs multicellulaires parallèle}

2.1. Introduction. - Les contraintes en termes de puissance commutée et de fréquence de découpage imposées par certains cahiers des charges imposent souvent au concepteur l'utilisation d'association d'interrupteurs car les caractéristiques des composants de l'électronique de puissance s'avèrent alors insuffisantes.

Pour les structures dites à fort courant, la mise en parallèle peut être effectuée à deux niveaux différents :

- Au niveau des composants. L'équilibrage des courants est obtenu par un système complexe de compensation des différentes caractéristiques des composants [8,9]. De plus, dans certains cas, le coefficient de température négatif de la tension de saturation nécessite un excellent refroidissement et un bon couplage thermique afin d'éviter tout risque d'emballement thermique. Enfin malgré l'augmentation du nombre d'interrupteurs, les formes d'ondes en sortie ne sont pas améliorées puisqu'il n'y a pas de degrés de liberté supplémentaires.

- Au niveau des cellules de commutation. Ici, l'équilibrage des courants est obtenu par un asservissement de chaque cellule. Ce type d'association autorise un entrelacement des commandes des diverses cellules mais impose leur connexion at moyen d'inductances destinées à supporter les différences entre les tensions instantanées des diverses cellules.

Les convertisseurs multicellulaires parallèles, éléments de cette deuxième catégorie ont donc l'avantage d'améliorer le spectre du courant de sortie mais sans nécessiter de contrôle des courants dans chaque cellule puisque l'équilibrage de ceux-ci est assuré par un dimensionnement judicieux du filtre d'entrée.

2.2. Topologie ET PRincipe de fonctionnement. - La mise en parallèle de cellules de commutation s'effectue en connectant directement les cellules aux bornes de la source de tension et en connectant leurs points milieu par le biais d'inductances de liaison comme le montre la figure 1.

Les deux interrupteurs d'une cellule élémentaire obéissent aux lois régissant le fonctionnement d'une cellule de commutation classique :

- les deux interrupteurs ne doivent jamais être passants simultanément car cela entraînerait un court-circuit de la source de tension,

- les deux interrupteurs ne doivent jamais être bloqués simultanément car la source de courant $I_{\mathrm{s}}$ serait alors en circuit ouvert,

- en pratique, des temps morts devront être prévus,

- à partir de ce schéma, on peut réaliser tous les types de conversion $(\mathrm{DC} / \mathrm{DC}, \mathrm{DC} / \mathrm{AC}$, $\mathrm{AC} / \mathrm{AC})$,

- les réversibilités en courant et en tension des interrupteurs doivent être compatibles avec les réversibilités des sources de courant $I_{\mathrm{s}}$ et de tension $E$.

2.3. Contraintes En Courant. - L'intérêt de ce type de structure réside dans le fait que les sources intermédiaires permettent de maîtriser la répartition des courants dans les interrupteurs passants. En effet, la loi des nœuds appliquée à chaque cellule de commutation élémentaire permet de montrer que :

$$
\left(I_{\mathrm{tr}_{k}}\right)_{\mathrm{on}}=I_{L_{k}} \text { avec } k \in\{1, \ldots, p\} .
$$




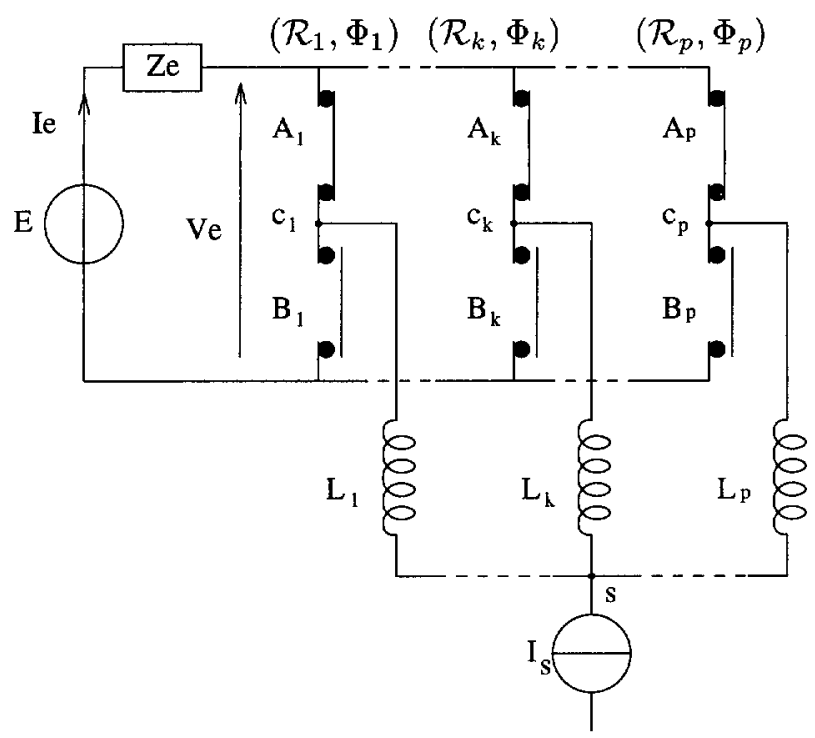

Fig. 1. - Topologie d'un bras multicellulaire parallèle.

[Topology of parallel multicell inverter leg]

Cette structure devient donc particulièrement intéressante si la répartition des courants se fait de manière équilibrée, c'est-à-dire :

$$
I_{L_{k}}=\frac{I_{\mathrm{s}}}{p} \Longrightarrow\left(I_{\mathrm{tr}_{\mathrm{k}}}\right)_{\mathrm{on}}=\frac{I_{\mathrm{s}}}{p} \text { avec } k \in\{1, \ldots, p\}
$$

2.4. Contraintes en tension. - La tension instantanée aux bornes de chaque interrupteur est égale à la tension d'entrée $V_{\mathrm{e}}$ lorsqu'il est bloqué et à zéro lorsqu'il est passant. Par conséquent, si $V_{\mathrm{e}}$ est assimilée à une source continue sur une période de découpage, la tension moyenne au point milieu $c_{k}$ de la cellule de commutation $k$ vaut :

$$
V_{c_{k}}=\mathcal{R}_{k} V_{\mathrm{e}} \text { avec } k \in\{1, \ldots, p\} .
$$

Pour obtenir des courants stables (sans même parler de courants équilibrés), il faut que les tensions moyennes aux bornes des inductances soient nulles, ce qui nécessite donc des rapports cycliques égaux.

2.5. AnAlyse du Courant DE sortie. - Les études menées sur les convertisseurs multicellulaires série ont montré que pour un déphasage de $2 \pi / p$ des signaux de commande des cellules de commutation, les premiers harmoniques de la tension de sortie étaient repoussés à $p$ fois la fréquence de découpage.

Le schéma présenté figure 2 permet de montrer dans le cas de trois cellules que l'on obtient une réduction intéressante de l'ondulation du courant de sortie dans un convertisseur multicellulaire parallèle à condition de déphaser les signaux de commande de $2 \pi / 3$.

De plus, nous verrons dans le chapitre suivant que le dispositif de rééquilibrage naturel des courants dans les inductances de liaison ne fonctionne que pour un déphasage de $2 \pi / p$. 

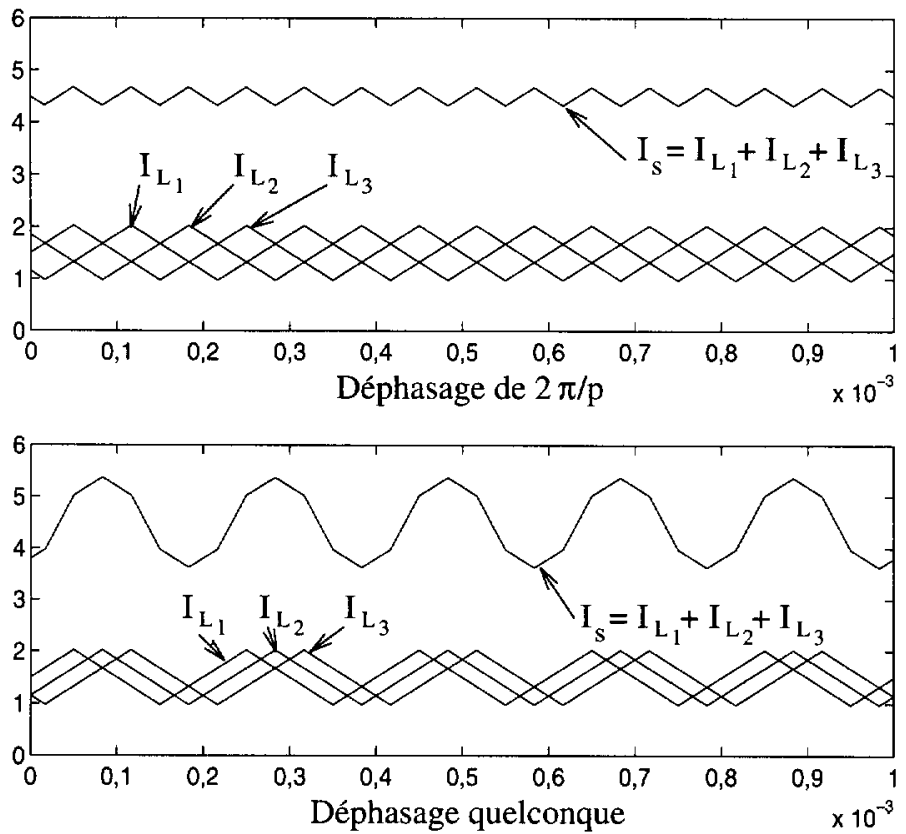

Fig 2 - Formes d'ondes du courant de sortie.

[Output current waveforms ]

\section{Stabilité de la répartition des courants}

3.1. Position du PROBlÈme. - La survie du convertisseur dépendant de la répartition des courants interrupteurs, il ne suffit pas de vérifier que le fonctionnement décrit ci-dessus est viable, il faut également s'assurer de sa stabilité, c'est-à-dire déterminer si le système est capable de rétablir l'équilibre à partir d'un état différent. À ce stade de l'étude, nous pouvons juste énoncer les conclusions suivantes :

- si les rapports cycliques sont égaux et la tension $V_{\mathrm{e}}$ est rigoureusement continue, chaque inductance de liaison voit une tension moyenne nulle à ses bornes et son courant moyen sur une période de découpage est donc constant quel que soit l'état initial,

- si le déphasage des commandes est de $2 \pi / p$ le spectre harmonique du courant de sortie est optimisé.

3.2. RÉÉquilibrage DES COURANTS POUR DEUX CEllules. - Sur la figure 1 nous avons mentionné l'existence d'une impédance $Z_{\mathrm{e}}$ en série avec la source de tension d'entrée. Le rôle de cette impédance est primordial, puisque comme nous allons le volr, c'est par un choix judicieux de sa valeur qu'un rééquilibrage naturel des courants dans les interrupteurs passants sera assuré.

Pour comprendre ce phénomène, nous allons illustrer l'influence de cette impédance dans le cas de deux cellules en parallèle (Fig 3 ).

Lorsque l'on a un déséquilibre des courants, il existe un harmonique à la fréquence de découpage sur le courant d'entrée $\left(I_{\mathrm{e}}^{1}\right)$. Par l'intermédiaire de l'impédance $Z_{\mathrm{e}}$, cet harmonique de 

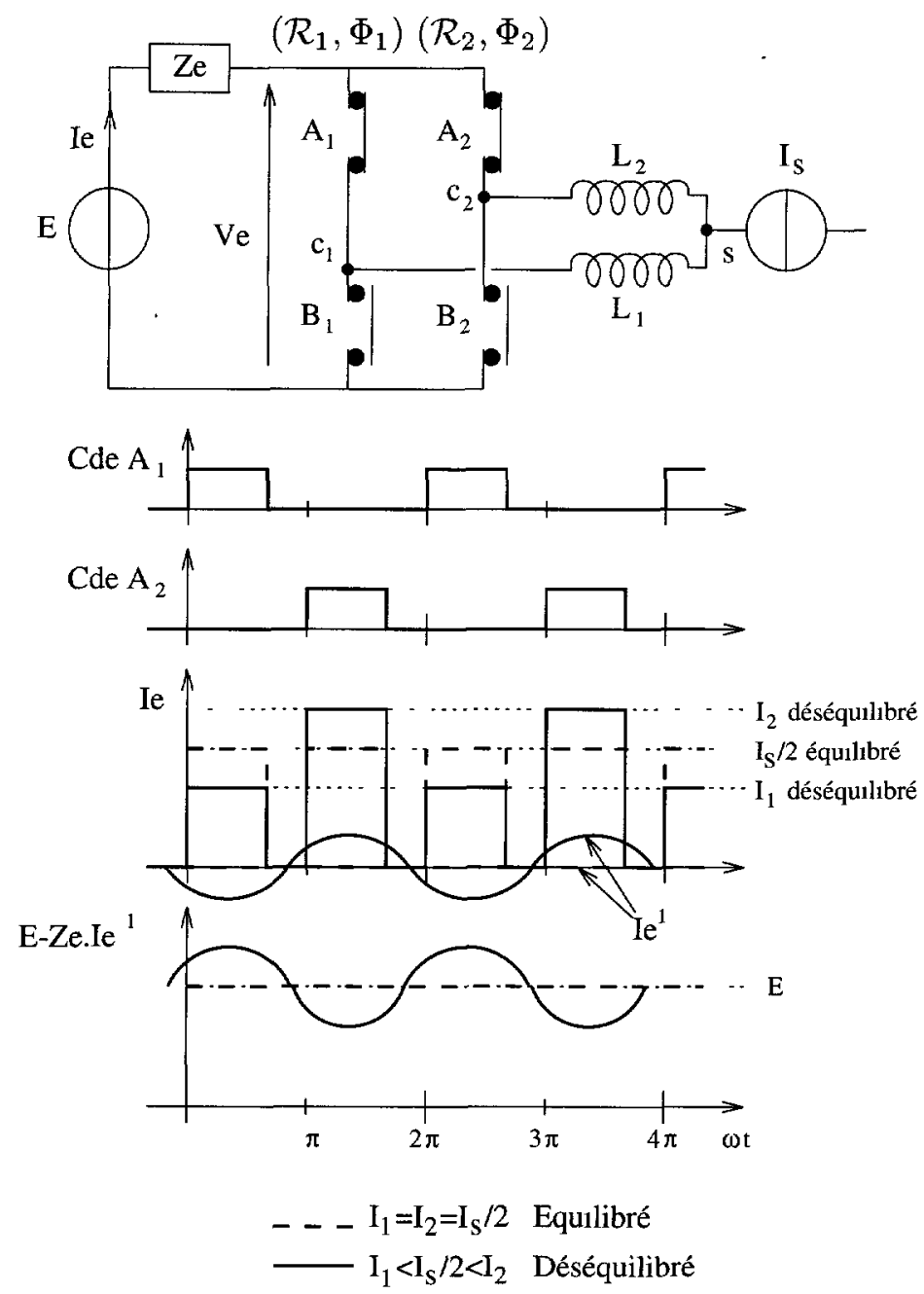

Fig. 3 - Rééquilibrage naturel pour 2 cellules.

[Natural balancing for 2 cells.]

courant introduit une modulation de la tension $V_{\mathrm{e}}$ de sorte que

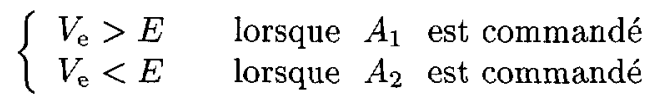

Bien que l'on ait égalité des rapports cycliques, on a donc:

$$
V_{\mathrm{c}_{1}}>\mathcal{R} E \text { et } V_{\mathrm{c}_{2}}<\mathcal{R} E \text {. }
$$

Par conséquent, le courant $I_{L_{1}}$ aura tendance à croître et $I_{L_{2}}$ à décroítre, ce qui correspond à un rééquilibrage automatique des courants.

Lorsque ces courants sont équilibrés, l'harmonique du courant d'entrée à la fréquence de découpage disparaît et il n'y a plus de modulation de la tension d'entrée à cette fréquence. 


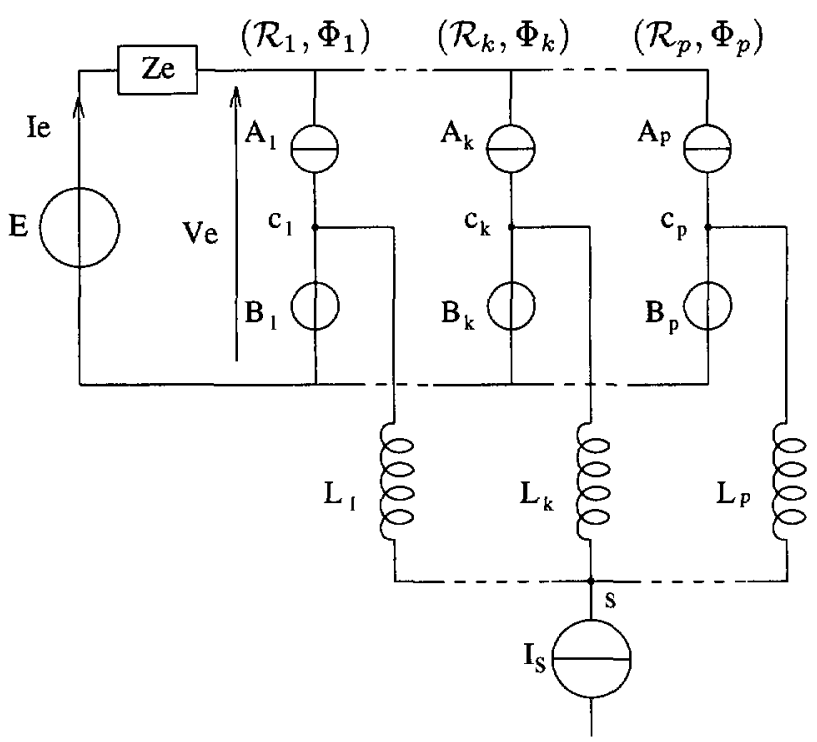

Fig. 4 - Configuration adoptée pour la modélisation

[Structure used for modelling.]

Donc, pour que $Z_{\mathrm{e}}$ ne dissipe pas de puissance en régime permanent, il faut que la partie réelle de son impédance soit nulle pour les fréquences présentes en régime équilibré, c'est-à-dire pour $2 F_{\text {dec }}(\mathrm{HF})$ et la fréquence de modulation du rapport cyclique (B.F.) en fonctionnement onduleur.

3.3 GÉnéralisation À $p$ CELlules. - En généralisant ce résultat à $p$ cellules en parallèle, nous pouvons déduire qu'à l'équilibre, le courant d'entrée ne possède que des harmoniques de rang multiple du nombre de cellules. À ces fréquences, la partie réelle de l'impédance $Z_{\mathrm{e}}$ doit donc être la plus faible possible pour limiter les pertes. Par contre, en cas de déséquilibre il apparaît des harmoniques de courant à $F_{\mathrm{dec}}, 2 F_{\mathrm{dec}}, \ldots,(p-1) F_{\mathrm{dec}}$. À ces fréquences, l'impédance du filtre doit être non négligeable afin de générer des harmoniques de tension et d'obtenir un bon rééquilibrage.

3.4. Conclusion. - La principale conclusion à tirer du paragraphe précédent est que le rééquilibrage est lié à des harmoniques de courant générés par le convertisseur. Le but de cet article est de développer un modèle du circuit qui tient compte de ces harmoniques afin de représenter et de quantifier le rééquilibrage pour un nombre quelconque de cellules.

\section{Modélisation}

De manière générale, l'étude d'un convertisseur peut être ramenée à celle d'un circuit obtenu en remplaçant pour une configuration quelconque de fonctionnement, les interrupteurs passants par des sources de courants représentant leurs courants et les interrupteurs bloqués par des sources de tensions représentant leurs tensions. Pour le convertisseur de la figure 1 et en considérant tous les interrupteurs de la rangée inférieure bloqués, l'étude est ramenée au circuit de la figure 4. 
4.1. HypothèsEs. - Les hypothèses nécessaires à la mise en équation sont les suivantes :

- les interrupteurs sont idéalisés (tension de saturation, courant de fuite et temps de commutation nuls),

- les interrupteurs étant parfaits, les temps morts sont supposés nuls,

- les inductances de liaison des cellules sont dimensionnées pour limiter les variations du courant commuté par chaque cellule ; ces courants seront supposés constants sur une période de découpage,

- de plus, les variations des courants moyens des cellules seront supposées lentes vis-à-vis de la fréquence de découpage ainsi que les celles du courant de sortie $I_{5}$,

- la source d'alimentation $E$ est supposée continue.

4.2. PRINCIPE DU CALCUL. - La modélisation présentée en annexe est effectuée en considérant les harmoniques de la fréquence de découpage à $r$ fois la fréquence de découpage selon la procédure suivante :

- le système étant dans un état donné, la connaissance des signaux de commande sur une période de découpage complète $\left(\mathcal{R}_{k}\right.$ et $\Phi_{k}$ avec $\left.k \in\{1, \ldots, p\}\right)$ permet de déterminer la phase et l'amplitude des harmoniques de courant dans les interrupteurs $A_{k}$,

- le courant d'entrée du bras est la somme des courants dans les interrupteurs $A_{k}$ et l'on obtient donc facilement les harmoniques de $I_{\mathrm{e}}\left(I_{\mathrm{e}}^{1}, I_{\mathrm{e}}^{2}, \ldots, I_{\mathrm{e}}^{r}\right.$ complexes),

- en fonction des caractéristiques du filtre d'entrée $\left(Z_{e}\right.$, complexe), ces harmoniques de courant donnent naissance à des harmoniques de tension $\left(V_{\mathrm{e}}^{1}, V_{\mathrm{e}}^{2}, \ldots, V_{\mathrm{e}}^{r}\right.$ complexes),

- la tension moyenne aux bornes des interrupteurs $B_{k}$ peut être calculée en fonction du déphasage de sa commande par rapport à la tension d'entrée,

- en tenant compte des paramètres de la charge $\left(R_{\mathrm{ch}}, L_{\mathrm{ch}}, V_{\mathrm{ch}}\right)$ et des valeurs des inductances de liaison $\left(L_{k}\right)$, les variations moyennes du courant dans les inductances de liaison $\left(\dot{I}_{L_{k}}\right)$ peuvent alors être déduites.

L'utilisation de cette procédure nous conduit à un modèle de représentation de la forme :

$$
\left[\begin{array}{c}
\dot{I}_{L_{1}} \\
\vdots \\
\dot{I}_{L_{p}}
\end{array}\right]=\tilde{A}_{\mathrm{ch}}\left[\begin{array}{c}
I_{L_{1}} \\
\vdots \\
I_{L_{p}}
\end{array}\right]+\tilde{B}_{\mathrm{ch}}\left[\begin{array}{c}
V_{\mathrm{ch}} \\
E
\end{array}\right]
$$

\section{Validation du modèle}

Le modèle ainsi généré permet de connaître l'évolution des courants dans les inductances de liaison à partir de conditions initiales quelconques et pour une combinaison de rapport cyclique donnée. Nous proposons ci-après de valider ce modèle en utilisant un outil de simulation prenant en compte la structure du convertisseur. Cette validation se fera sur un convertisseur de type hacheur dévolteur à quatre cellules en parallèle dont les caractéristiques sont données figure 5 .

Nous avons délibérément choisi les éléments du convertisseur de manière à ne pas avoir un fonctionnement optimisé et ainsi pouvoir observer les déséquilibres des courants mais aussi pour 


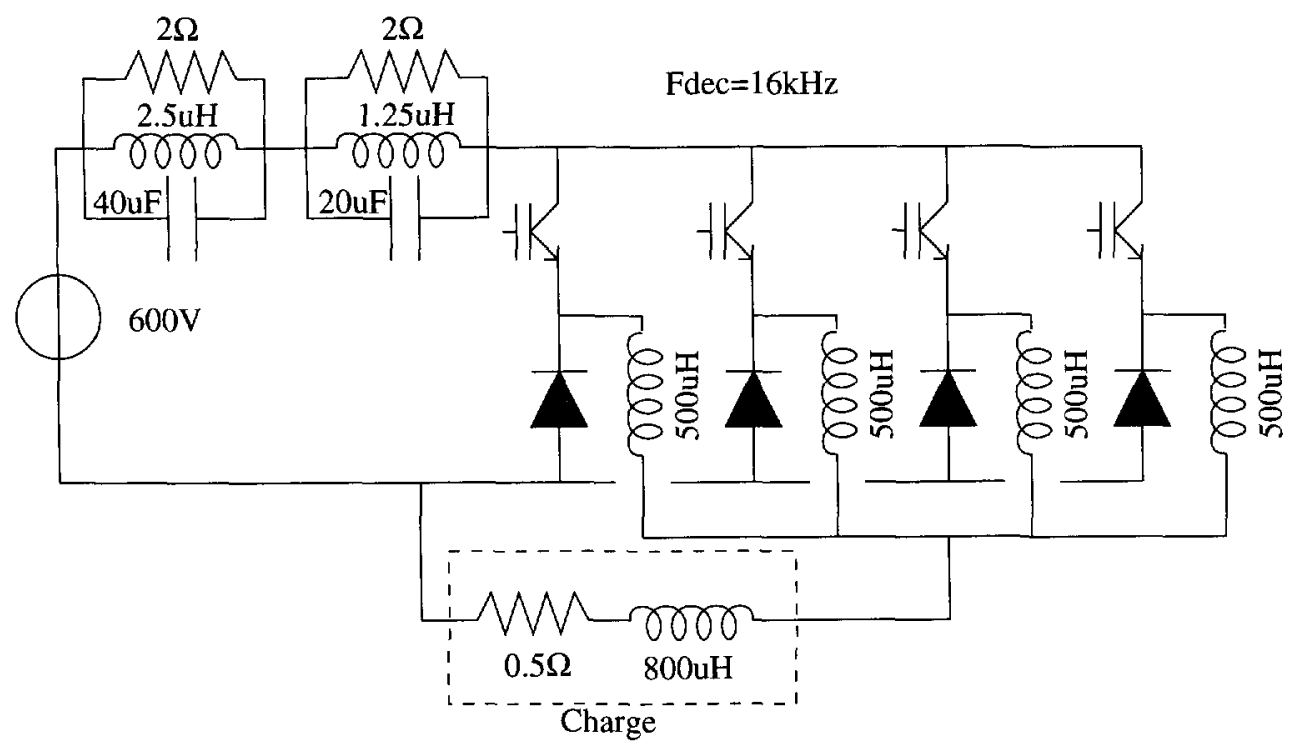

Fig 5 - Caractéristiques du hacheur dévolteur utılisé pour la valıdation.

[Characteristics of the buck used for the validation.]

valider le modèle dans des conditıons extrêmes de fonctionnement. Pour les mêmes raisons, les perturbations provoquées (erreur sur les rapports cycliques et les déphasages, $R_{\mathrm{ds}_{\mathrm{on}}}$ des interrupteurs, etc.) ont été volontairement exagérées.

Enfin, pour avoir une bonne précision du modèle, nous prendrons en compte les 32 premiers harmoniques. Au-delà, l'impédance d'entrée $Z_{e}$ est négligeable et par conséquent l'influence des harmoniques de rang supérieur est nulle.

5.1. Comparaison AVEC DES SIMUlations SUCCESS. - Le logiciel SUCCESS servira de référence pour notre comparaison et nous nous proposons d'évaluer la justesse et la précision du modèle pour différents cas de figures.

Les simulations présentées dans les figures suivantes montrent les courants dans chaque inductance de liaison pour la simulation du modèle (traits noirs) et de SUCCESS.

5.2. DÉmarrage avec une erreur Sur les dÉPhasages Puis CORRECtion. - Dans cette simulation, nous pouvons noter deux phases distinctes:

- $0<t<3 \mathrm{~ms}$ : le déphasage par rapport à la cellule 1 des autres cellules est de :

$108^{\circ}$ pour la cellule 2 ,

$189^{\circ}$ pour la cellule 3 ,

$252^{\circ}$ pour la cellule 4 .

- $3<t<15 \mathrm{~ms}$ : le déphasage par rapport à la cellule 1 des cellules 2,3 et 4 est respectivement de $90^{\circ}, 180^{\circ}$ et $270^{\circ}$.

Les résultats obtenus permettent de vérifier la vahdité du modèle par rapport aux déphasages des commandes. quelles soient parfaites ou perturbées. 


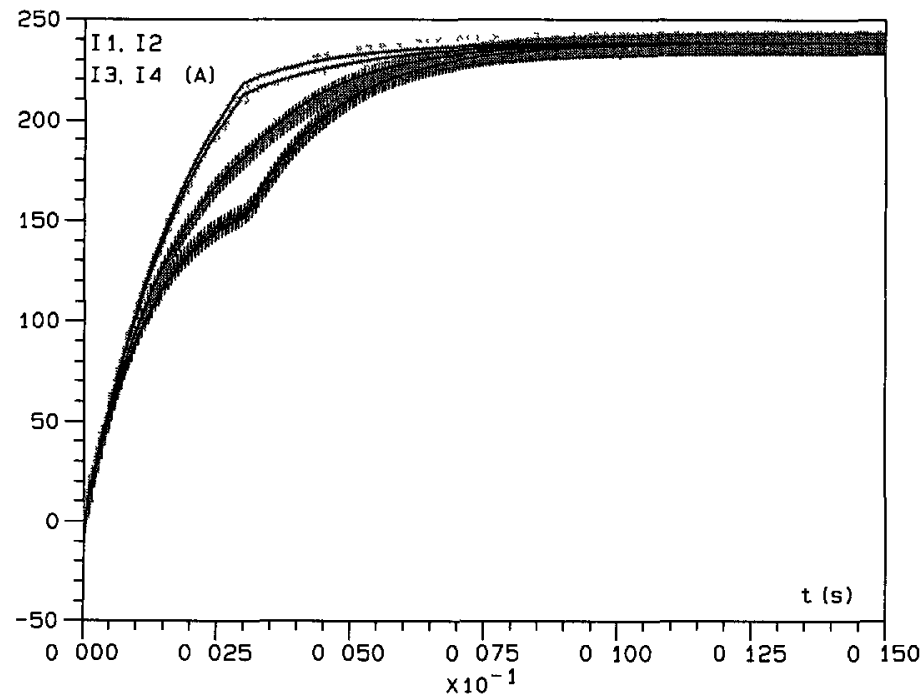

Fig 6. - Déséquilibre des déphasages · simulation et modèle.

[Unbalanced phase-shifts: simulation and model]

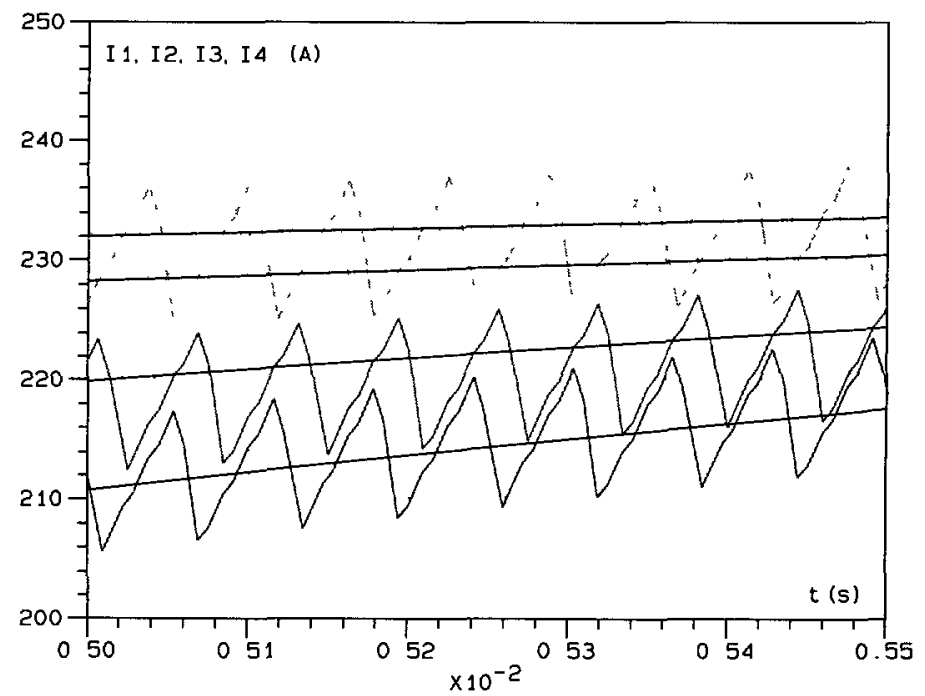

Fig. 7. - Agrandissement entre 5 et $5,5 \mathrm{~ms}$.

[Expand between 5 and 5.5 ms.]

En effectuant un agrandissement à l'échelle de quelques périodes de découpage de cette simulation (Fig. 7), il apparaît nettement que le modèle ne tient pas compte des ondulations du courant dans les inductances de liaisons mais qu'il correspond à la valeur moyenne de celui-ci. 


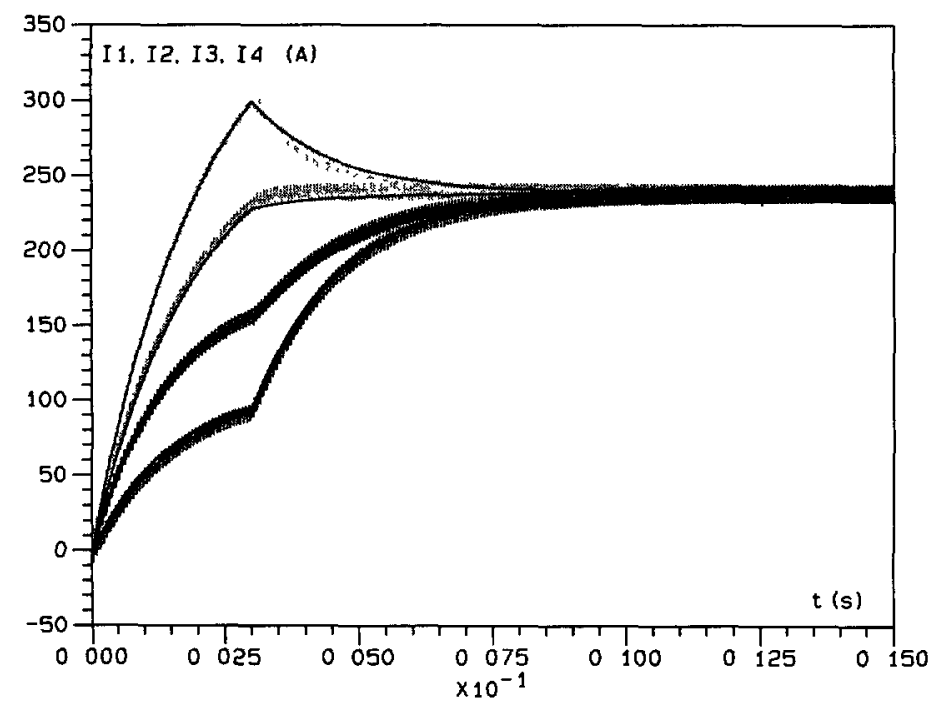

Fig. 8. - Déséquilibre des rapports cycliques : simulation et modèle

[Unbalanced duty cycles: simulation and model]

5.3. DÉmARRAGE AVEC UNE ERREUR SUR LES RAPPORTS CYCLIQUES PUIS CORRECTION. Dans cette simulation (Fig. 8), nous pouvons noter deux phases distinctes :

- $0<t<3 \mathrm{~ms}: \mathcal{R}_{k}=0,8$ dans les quatre cellules auquel nous avons rajouté une erreur de :

$+7 \%$ pour la cellule 1 ,

$+3 \%$ pour la cellule 2 ,

$0 \%$ pour la cellule 3 ,

$-5 \%$ pour la cellule 4 .

- $3<t<15 \mathrm{~ms}: \mathcal{R}_{k}=0,8$ dans les quatre cellules.

Les résultats obtenus valident la prise en compte des différents rapports cycliques dans le modèle qu'ils soient égaux ou bien perturbés.

5.4. DÉmarRage AVEC $R_{\text {ds }_{\text {on }}}$ DiffÉREnTES. - Les valeurs de $R_{\text {ds }_{\text {on }}}$ pour les quatre cellules sont les suivantes : $1,30,110$ et $200 \mathrm{~m} \Omega$.

Les résultats présentés figure 9 montrent que le modèle peut tenir compte des différentes $R_{\mathrm{ds}_{\text {on }}}$ des interrupteurs et que l'utilisation d'une impédance d'entrée adaptée permet de rédure les déséquilibres des courants.

5.5. DÉmarRage AVEC QUATRE CEllules PUIS ARRÊT DE L'UnE D'EnTRE Elles. - Cette simulation avec le modèle a dû être réalisée en deux fois car l'arrêt de la cellule 4 s'effectue en deux phases distinctes.

La première simulation comporte le démarrage avec un rapport cyclique de 0,8 pour les quatre cellules et un déphasage de $2 \pi / 4$. $\grave{A} t=3 \mathrm{~ms}$, on fixe le rapport cyclique de la cellule 4 à zéro pour simuler le blocage de l'interrupteur $A_{4}$. Le courant dans l'inductance $L_{4}$ décroît 


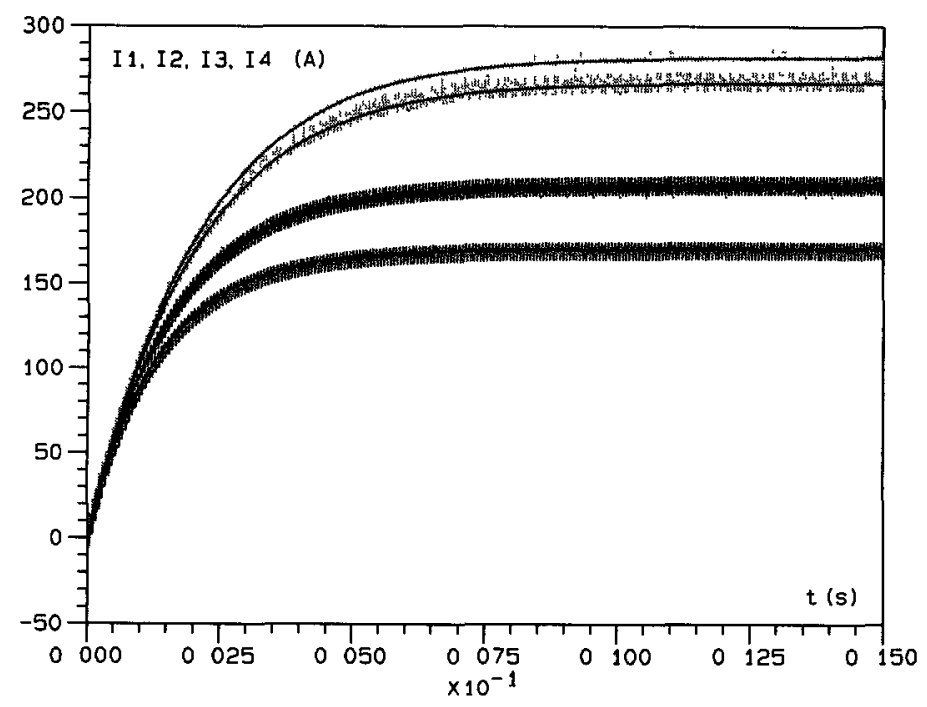

Fig. 9 - Différentes $R_{\mathrm{ds}_{\text {on }}}$ simulation et modèle [Different $R_{\mathrm{ds}_{\mathrm{on}}}$ : simulation and model.]

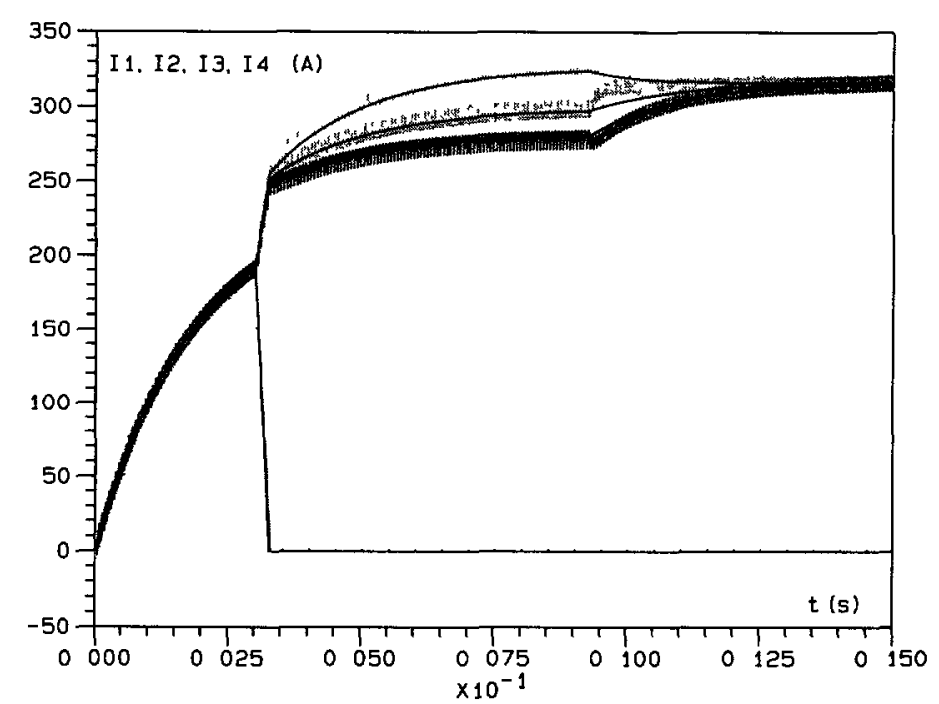

Fig 10. - Arrêt d'une cellule : simulation et modèle.

[Stop of one cell: simulation and model.]

car la diode de roue libre $\left(B_{4}\right)$ continue de conduire. $\grave{A} t=3,3 \mathrm{~ms}$, ce courant s'annule et la diode se bloque.

La seconde simulation commence alors avec les paramètres et les conditions finales de la première, mais avec seulement trois cellules de commutation. $\grave{A} t=9,3 \mathrm{~ms}$, on corrige le déphasage en le fixant à $2 \pi / 3$.

Dans cette situation encore, le modèle permet de prédire avec exactitude le comportement du convertisseur. 


\section{Utilité du modèle}

La mise à jour d'un modèle mathématique de représentation pour un convertisseur statique facilite l'analyse quantitative de son comportement. Malgré le caractère non-linéaire de ces convertisseurs, il devient simple d’extraire les caractéristiques essentielles qualifiant le régime statique et dynamique. Parmi les nombreuses utılisations liées à l'existence d'un tel modèle, notamment en termes d'analyse et de commande, nous proposons trois illustrations immédiates dont l'intérêt est certain.

6.1. DÉtermination directe DU RÉgime permanent. - Le modèle d'état du convertisseur (Éq. 6) représente l'évolution des courants dans les inductances de liaison en fonction des rapports cycliques. S'il est simple de calculer le régime d'équilibre lorsque les rapports cycliques sont tous égaux et les commandes déphasées de $2 \pi / p$, le problème est plus complexe pour une distrıbution quelconque de rapport cyclique. $\grave{A}$ ce niveau là, le modèle s'avère très utile, ainsı le point d'équilibre des courants dans les inductances de liaison représentées par $X$ atteint son régime permanent $X_{\mathrm{p}}$ déterminé par :

$$
\dot{X}=0 \Longrightarrow X_{\mathrm{p}}=-A^{-1} B\left[\begin{array}{c}
V_{\mathrm{ch}} \\
E
\end{array}\right]
$$

La connaissance de ces courants d'équilibre par la voie d'une simulation traditionnelle devient longue et fastidieuse notamment s1 l'on désire en faire l'étude en fonction des différents rapports cycliques.

6 2. EXTRACTION DES CONSTANTES DE TEMPS. - Une caractéristique importante tradusant la rapidité d'équilibrage des courants dans les inductances de liaison peut également être calculée par ce modèle de représentation. En effet pour un rapport cyclique fixé, toute la $\mathrm{dy}$ namique du dispositif est contenue dans la matrice $A$ dite matrice dynamique. Le calcul des valeurs propres de cette matrice permet de caractériser les modes du système c'est-à-dıre les constantes de temps.

Les constantes de temps ainsi déterminées nous renseignent sur la durée du transitoire et donc sur le temps de rééquilibrage des courants dans les inductances de liaison.

6.3. Diagramme de Bode. - Pour un fonctionnement à rapport cyclique constant, il est simple de déterminer la matrice de transfert liant l'évolution des courants dans les inductances de liaison aux grandeurs de la matrice de commande (tension d'alimentation $E$ et la f.e.m. de la charge $V_{\mathrm{ch}}$ ). En effet la transformée de Laplace de l'équation (Éq. 6) nous donne :

$$
T(p)=\left(p I_{p}-A\right)^{-1} B .
$$

L'analyse de ces transmittances permet de rassembler les deux résultats précédents et de positionner sur le plan fréquentiel les différentes pulsations de coupure.

\section{Conclusion}

La mise à jour d'un modèle mathématique représentant le comportement dynamique et statique d'un convertisseur multicellulaire parallèle ne peut être réalisé qu'en considérant des phénomènes rapides se déroulant à l'intérieur d'une période de découpage. Cette considération conduit à rejeter toute méthode de modélisation basée sur la représentation au sens des valeurs moyennes. À cet effet, la méthode développée dans cet article utilise une décomposition harmonique du courant circulant dans les interrupteurs par le biais d'une source de courant 


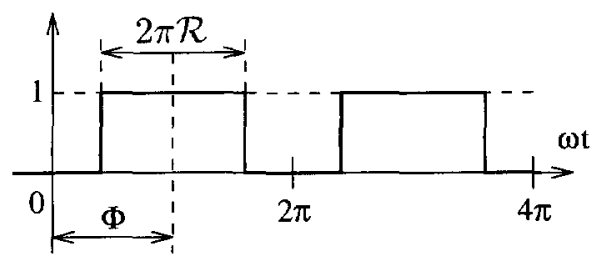

Fig. 11. - Définition des notations.

[Definition of symbols.]

équivalente. L'association de ces diverses sources conformément à la topologie du convertisseur étudié nous conduit à une équation d'état globale représentant l'évolution des courants dans les inductances de liaison. Cette relation prend en compte les différents rapports cycliques de chaque cellule et permet ainsi de représenter de manière fidèle le comportement du convertisseur. Au nombre des utilisations intéressantes de ce modèle nous avons noté la recherche du régıme d'équilibre ainsi que l'extraction des constantes de temps. La linéarisation de ce modèle autour d'un point de fonctionnement fait également partie des procédures intéressantes qui permettront de définir des lois de commande performantes.

\section{Annexe A}

\section{A. Calcul des éléments de la matrice dynamique}

A.1. Modèle PRENANT EN COMPTE L'harmonique de RANG $n$. - L'harmonique de rang $n$ d'un signal carré prenant les valeurs 0 ou 1 , de phase $\Phi$ et de rapport cyclique $\mathcal{R}$ (Fig. 11) est donné par :

$$
H_{n}=\frac{2}{n \pi} \sin (n \pi \mathcal{R}) \exp (j n \Phi)
$$

Le courant $I_{\mathrm{e}}$ fourni par la source $E$ ( $c f$. Fig. 4) peut s'exprimer comme la somme des courants circulant dans les interrupteurs de la rangée supérieure. De plus, l'interrupteur $A_{k}$ impose un courant nul lorsqu'il est bloqué et est parcouru par $I_{L_{k}}$ lorsqu'il est passant. Par conséquent $I_{e}^{n}$, l'harmonique de rang $n$ du courant fourni par la source :

$$
I_{\mathrm{e}}^{n}=\sum_{k=1}^{p} \frac{2}{n \pi} I_{L_{k}} \sin \left(n \pi \mathcal{R}_{k}\right) \exp \left(\jmath n \Phi_{k}\right) .
$$

En introduisant,

$$
G_{k}^{n}=\frac{1}{n \pi} \sin \left(n \pi \mathcal{R}_{k}\right) \exp \left(J n \Phi_{k}\right)
$$

l'équation (A.2) peut s'écrire sous la forme matricielle suivante :

$$
I_{\mathrm{e}}^{n}=2\left[G_{1}^{n} \cdots G_{p}^{n}\right]\left[\begin{array}{c}
I_{L_{1}} \\
\vdots \\
I_{L_{p}}
\end{array}\right]
$$




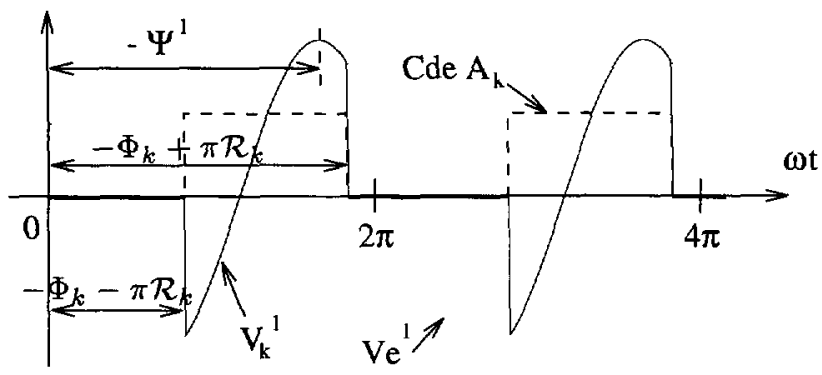

Fig. 12. - Influence d'un harmonique de la tension $V_{\mathbf{e}}$ sur le potentiel du noud $c_{k}$ : cas particulier de l'harmonique à $F_{\text {dec }}\left(V_{\mathrm{e}}^{1}\right)$

[Influence of one harmonic of voltage $V_{\mathrm{e}}$ on the potential at node $c_{k}$ : special case of the harmonic at $F_{\text {dec }}\left(V_{\mathrm{e}}^{1}\right)$.]

Compte tenu de l'hypothèse concernant les lentes variations de la valeur moyenne des courants $I_{k}$ et de l'équation (A.4), nous pouvons considérer que $I_{e}^{n}$ ne subit pas de fortes variations entre deux périodes de découpage ce qui nous permet alors d'exprimer l'harmonique de rang $n$ de la tension d'entrée en fonction du courant et de l'impédance d'entrée à cette fréquence .

$$
V_{\mathrm{e}}^{n}=-Z_{\mathrm{e}}^{n} I_{\mathrm{e}}^{n}=\left\|V_{\mathrm{e}}^{n}\right\| \exp \left(\jmath \Psi^{n}\right)
$$

Cette tension harmonique associée à la fonction de modulation de la cellule $k$ génère une tension au point $c_{k}$ que nous noterons $V_{k}^{n}$ qui dépend du rapport cyclique $\mathcal{R}_{k}$ et du déphasage $n \Phi_{k}-\Psi^{n}$ (Fig. 12).

La valeur moyenne de cette tension créée par l'harmonique de tension $n$ sur la cellule $k$ s'exprime par :

$$
\bar{V}_{k}^{n}=-\frac{1}{2 \pi} \int_{-\Phi_{k}-\pi \mathcal{R}_{k}}^{-\Phi_{k}+\pi \mathcal{R}_{k}}\left\|V_{\mathrm{e}}^{n}\right\| \cos \left(n \Theta+\Psi^{n}\right) \mathrm{d} \Theta
$$

Ce qui donne :

$$
\bar{V}_{k}^{n}=-\frac{\left\|V_{\mathrm{e}}^{n}\right\|}{n \pi} \sin \left(n \pi \mathcal{R}_{k}\right) \cos \left(n \Phi_{k}-\Psi^{n}\right) .
$$

Cette quantité peut être obtenue sous la forme :

$$
\bar{V}_{k}^{n}=\Re\left\{\frac{1}{n \pi} \sin \left(n \pi \mathcal{R}_{k}\right) \exp \left(-\jmath n \Phi_{k}\right)\left\|V_{\mathrm{e}}^{n}\right\| \exp \left(\jmath \Psi^{n}\right)\right\} .
$$

Et, compte tenu de la grandeur introduite plus haut (Éq. (A.3)), on a alors :

$$
\bar{V}_{k}^{n}=\Re\left\{\left(G_{k}^{n}\right)^{*} V_{\mathrm{e}}^{n}\right\} .
$$

L'inductance $L_{k}$ est soumise à trois influences principales ; la tension $E$ appliquée avec un rapport cyclique $\mathcal{R}_{k}$, le $n$-ième harmonique de tension calculé ci-dessus et le potentiel $V_{\mathrm{s}}$. On a donc :

$$
\dot{I}_{L_{k}}=\frac{1}{L_{k}} V_{L_{k}}=\frac{1}{L_{k}}\left[\mathcal{R}_{k} E+\bar{V}_{k}^{n}-V_{\mathrm{s}}\right]
$$


On peut l'écrire sous la forme matricielle suivante :

$$
\left[\begin{array}{c}
\dot{I}_{L_{1}} \\
\vdots \\
\dot{I}_{L_{p}}
\end{array}\right]=\Re\left\{\left[\begin{array}{c}
\frac{1}{L_{1}}\left(G_{1}^{n}\right)^{*} \\
\vdots \\
\frac{1}{L_{p}}\left(G_{p}^{n}\right)^{*}
\end{array}\right] V_{\mathrm{e}}^{n}\right\}+\left[\begin{array}{cc}
-\frac{1}{L_{1}} & \frac{\mathcal{R}_{1}}{L_{1}} \\
\vdots & \vdots \\
-\frac{1}{L_{p}} & \frac{\mathcal{R}_{p}}{L_{p}}
\end{array}\right]\left[\begin{array}{c}
V_{\mathrm{s}} \\
E
\end{array}\right]
$$

À partir de (A.4), (A.5) et (A.11), on obtient alors :

$$
\left[\begin{array}{c}
\dot{I}_{L_{1}} \\
\vdots \\
\dot{I}_{L_{p}}
\end{array}\right]=-2 \Re\left\{Z_{\mathrm{e}}^{n}\left[\begin{array}{c}
\frac{1}{L_{1}}\left(G_{1}^{n}\right)^{*} \\
\vdots \\
\frac{1}{L_{p}}\left(G_{p}^{n}\right)^{*}
\end{array}\right]\left[G_{1}^{n} \cdots G_{p}^{n}\right]\right\}\left[\begin{array}{c}
I_{L_{1}} \\
\vdots \\
I_{L_{p}}
\end{array}\right]+\left[\begin{array}{cc}
-\frac{1}{L_{1}} & \frac{\mathcal{R}_{1}}{L_{1}} \\
\vdots & \vdots \\
-\frac{1}{L_{p}} & \frac{\mathcal{R}_{p}}{L_{p}}
\end{array}\right]\left[\begin{array}{c}
V_{\mathrm{s}} \\
E
\end{array}\right]
$$

A.2. Modìle PREnANT En COMPte Plusieurs harmoniques. - En tenant compte des $r$ premiers harmoniques, l'équation (A.10) devient :

$$
\dot{I}_{L_{k}}=\frac{1}{L_{k}} V_{L_{k}}=\frac{1}{L_{k}}\left[\mathcal{R}_{k} E+\sum_{k=1}^{r} \bar{V}_{k}^{n}-V_{\mathrm{s}}\right] .
$$

Cette dernière relation peut s'écrire sous la forme matricielle suivante :

$$
\left[\begin{array}{c}
\dot{I}_{L_{1}} \\
\vdots \\
\dot{I}_{L_{p}}
\end{array}\right]=A\left[\begin{array}{c}
I_{L_{1}} \\
\vdots \\
I_{L_{p}}
\end{array}\right]+B\left[\begin{array}{c}
V_{\mathrm{s}} \\
E
\end{array}\right]
$$

Avec :

$$
\begin{aligned}
& A=-2 \Re\left\{\left[\begin{array}{ccc}
\frac{1}{L_{1}}\left(G_{1}^{1}\right)^{*} & \cdots & \frac{1}{L_{1}}\left(G_{1}^{r}\right)^{*} \\
\vdots & \ddots & \vdots \\
\frac{1}{L_{p}}\left(G_{p}^{1}\right)^{*} & \cdots & \frac{1}{L_{p}}\left(G_{p}^{r}\right)^{*}
\end{array}\right]\left[\begin{array}{ccc}
Z_{\mathrm{e}}^{1} G_{1}^{1} & \cdots & Z_{\mathrm{e}}^{1} G_{p}^{1} \\
\vdots & \ddots & \vdots \\
Z_{\mathrm{e}}^{r} G_{1}^{r} & \cdots & Z_{\mathrm{e}}^{r} G_{p}^{r}
\end{array}\right]\right\} \\
& B=\left[\begin{array}{cc}
-\frac{1}{L_{1}} & \frac{\mathcal{R}_{1}}{L_{1}} \\
\vdots & \vdots \\
-\frac{1}{L_{p}} & \frac{\mathcal{R}_{p}}{L_{p}}
\end{array}\right] .
\end{aligned}
$$

A.3. Modèle PREnANT En COMPte LA CHARGe du CONVERTisseur. - Le potentiel au point $\mathrm{s}$ peut évoluer par sa dynamique propre (f.e.m. notée $V_{\mathrm{ch}}$ ) mais il dépend aussi du courant imposé par le bras (par le biais de l'impédance $R_{\mathrm{ch}}$ et $L_{\mathrm{ch}}$ ). Ainsi l'on peut écrire :

$$
V_{\mathrm{s}}=V_{\mathrm{ch}}+R_{\mathrm{ch}} \sum_{k=1}^{p} I_{L_{k}}+L_{\mathrm{ch}} \sum_{k=1}^{p} \dot{I}_{L_{\alpha}} .
$$

Le système régit par l'équation (A.14) peut alors s'écrire sous la forme suivante :

$$
\begin{aligned}
{\left[\begin{array}{c}
\dot{I}_{L_{1}} \\
\vdots \\
\dot{I}_{L_{p}}
\end{array}\right] } & =A_{\mathrm{ch}}\left[\begin{array}{c}
I_{L_{1}} \\
\vdots \\
I_{L_{p}}
\end{array}\right]+B_{\mathrm{ch}}\left[\begin{array}{c}
V_{\mathrm{ch}} \\
E
\end{array}\right] \\
& =M^{-1}\left(A-R_{\mathrm{ch}}\left[\begin{array}{ccc}
\frac{1}{L_{1}} & \cdots & \frac{1}{L_{1}} \\
\vdots & \ddots & \vdots \\
\frac{1}{L_{p}} & \cdots & \frac{1}{L_{p}}
\end{array}\right]\right)\left[\begin{array}{c}
I_{L_{1}} \\
\vdots \\
I_{L_{p}}
\end{array}\right]+M^{-1} B\left[\begin{array}{c}
V_{\mathrm{ch}} \\
E
\end{array}\right]
\end{aligned}
$$


avec :

$$
M=[1]+L_{\mathrm{ch}}\left[\begin{array}{ccc}
I_{L_{1}} & \cdots & I_{L_{1}} \\
\vdots & \ddots & \vdots \\
I_{L_{j}} & \cdots & I_{L_{p}}
\end{array}\right]
$$

A.4. Modèle prenant en COMPte Les $R_{\mathrm{ds}_{o n}}$ Des interrupteurs - Pour la modélisation des interrupteurs, nous avons considéré que les deux interrupteurs d'une cellule $k$ avait la même $R_{\mathrm{ds}_{\mathrm{on}}}$ notée $R_{\mathrm{on}_{\Lambda}}$. Ainsi, leur prise en compte se résume à insérer une résistance en série avec l'inductance de liaison $L_{k}$.

Cette modification influe sur l'équation A.10 qui devient :

$$
\dot{I}_{L_{k}}=\frac{1}{L_{k}} V_{L_{k}}=\frac{1}{L_{k}}\left[\mathcal{R}_{k} E+\bar{V}_{k}^{n}-V_{\mathrm{s}}\right]-\frac{R_{\mathrm{on}_{k}}}{L_{k}} I_{L_{k}} .
$$

Le système global est donc régi par des équations déduites de (A.16) avec les modifications dues à (A.17), et par conséquent :

$$
\begin{aligned}
{\left[\begin{array}{c}
\dot{I}_{L_{1}} \\
\vdots \\
\dot{I}_{L_{p}}
\end{array}\right] } & =\tilde{A}_{\mathrm{ch}}\left[\begin{array}{c}
I_{L_{1}} \\
\vdots \\
I_{L_{p}}
\end{array}\right]+\tilde{B}_{\mathrm{ch}}\left[\begin{array}{c}
V_{\mathrm{ch}} \\
E
\end{array}\right] \\
& =\left(A_{\mathrm{ch}}-M^{-1}\left[\begin{array}{ccc}
\frac{R o n_{1}}{L_{1}} & & 0 \\
& \ddots & \\
0 & & \frac{R o n_{p}}{L_{p}}
\end{array}\right]\right)\left[\begin{array}{c}
I_{L_{1}} \\
\vdots \\
I_{L_{p}}
\end{array}\right]+B_{\mathrm{ch}}\left[\begin{array}{c}
V_{\mathrm{ch}} \\
E
\end{array}\right] .
\end{aligned}
$$

\section{Bibliographie}

[1] Middelbrook R.D. and Cuk S., A general unffied approach to modellıng switching converter power stages, IEEE PESC Record (1976) 18-34.

[2] Guillaud X. et Hautier J.P., Concepts de modélisation pour la commande des convertisseurs statiques, J. Phys. III France 4 (1994) 805-819.

[3] Bordry F., Synthèse des méthodes de simulation des convertisseurs statiques, Thèse de doctorat (INPT, 1985).

[4] Sira-Ramırez H., Sliding motions in bilinear switched networks, IEEE Trans. Crrcuits Systems (August 1987) 919-933.

[5] Vorperian V. and Cuk S., Small signal analysis of resonnant converter, IEEE PESC Record (1983) 269-282

[6] Collomb C., Fadel M. and Chéron Y., Analysis of dc/dc series resonnant converter operating above natural frequency, EPE 4 (Firenze, 1991) 542-547

[7] Sanders S., Noworolski J . Liu X. and Verghese G., Generalized averagıng method for power conversion circuits, IEEE PESC Record (1990) 333-340.

[8] Dumolard B., Contrôler huit gros igbts en parallèle, Rev. Electron. (août, 1994).

[9] Letor R., Static and dynamic behavior paralleled igbts. IEEE IAS Record (1990) 1604-1611.

[10] Meynard T A. and Davancens P., Current balance in paralleled commutation cells, PCIM 30 (Nurnberg, 1995) 127-133.

[11] Meynard T.A., Dıspositif d'équilıbrage des courants dans les associations parallèles de cellules de commutation, Brevet Français $N^{\circ} 94.09793$ (France, août 1994). 